\title{
Ghost poles in the nucleon propagator: Vertex corrections and form factors
}

\author{
G. Krein \\ Instituto de Física Teórica, Universidade Estadual Paulista, \\ Rua Pamplona, 145-01405 São Paulo, Brazil \\ M. Nielsen \\ Instituto de Física, Universidade de São Paulo, Caixa Postal, 20516-01498 São Paulo, Brazil \\ R. D. Puff and L. Wilets \\ Department of Physics, FM-15, University of Washington, Seattle, Washington 98195
}

(Received 23 December 1992)

\begin{abstract}
Vertex corrections are taken into account in the Schwinger-Dyson equation for the nucleon propagator in a relativistic field theory of fermions and mesons. The usual Hartree-Fock approximation for the nucleon propagator is known to produce the appearance of complex (ghost) poles which violate basic theorems of quantum field theory. In a theory with vector mesons there are vertex corrections that produce a strongly damped vertex function in the ultraviolet. One set of such corrections is known as the Sudakov form factor in quantum electrodynamics. When the Sudakov form factor generated by massive neutral vector mesons is included in the Hartree-Fock approximation to the Schwinger-Dyson equation for the nucleon propagator, the ghost poles disappear and consistency with basic requirements of quantum field theory is recovered.
\end{abstract}

PACS number(s): 21.30.+y, 21.60.Jz, 21.65.+f

\section{INTRODUCTION}

Nonrelativistic many-body theory has been used with success in the study of ground and excited states of nuclear systems. Nevertheless, experiments in the near future will provide data on nuclear systems at extreme conditions of density and temperature and obviously a new theoretical approach that goes beyond the nonrelativistic one will be required. This has motivated a great deal of interest in recent years in the development of relativistic many-body theories for nuclear physics based on the methods of renormalizable relativistic quantum field theory. In this context, there is an extensive literature on calculations of nuclear matter and finite nuclei properties by means of models based on the original Walecka model [1]. Calculations employing mean field and oneloop (Hartree) approximations have achieved considerable success in the description of bulk properties of nuclei and of proton-nucleus scattering parameters. However, there are severe difficulties in extending the calculations to include quantum corrections which go beyond the one-loop Hartree approximation. The inclusion of these quantum corrections leads to catastrophic results, with the appearance of complex poles in the baryon and meson propagators which, among other things, introduce a large imaginary part to the nuclear matter energy.

Complex poles, or ghosts, have long been noted in local relativistic field theory $[2,3]$. They are physically unacceptable because they correspond to eigenstates of the system with complex energies and probabilities. In the case of quantum electrodynamics (QED), the appearance of a ghost in the one-loop correction to the photon propagator, the so-called Landau ghost, is not taken as a serious drawback of the theory. This is because the momentum scale at which the ghost appears is far from measurable and, at this scale QED should probably be modified to include the effects of other electroweak effects. In the case of a nuclear theory with mesons and baryons, the corresponding ghosts appear at momentum scales of the order of $1 \mathrm{GeV}$. The appearance of the ghost poles is related to the short distance behavior of the model interactions; asymptotically free theories appear to be free of ghosts [4]. It is clear that a description of hadronic matter in terms of mesons and baryons only must break down in the region where short distance properties are involved. However, such a description should provide a reasonable description of the properties which are thought to be insensitive to the short distance physics. In this sense, the appearance of ghosts in hadronic theories appears to frustrate the hope of constructing relativistic models based on renormalizable field theories without employing subnucleonic degrees of freedom. Achtzehnter and Wilets [5] have shown that quark substructure plays an important role in the interaction of nucleons with an external (Bose) field at all momentum transfers. This involves issues which we will not pursue here. Rather, we concentrate on the construction of some intrinsically consistent field theory. Of course one should keep in mind that the effective Lagrangians commonly used in nuclear physics are likely not be derivable from the fundamental theory of the strong interactions (QCD). Such an effective Lagrangian will probably be very complicated and quite inelegant.

In the past, several methods have been proposed to eliminate this short distance sensitivity as, for example, modifying the analytic structure of the propagators 
in such a way to remove the unwanted singularities [6]. More recently, in the light of the quark substructure of the nucleons, form factors at the meson-baryon vertices [7] have been used to regulate the theory at short distances. Another perspective on the problem is the regulation of the theory by means of vector meson dressing of nucleon-meson vertices. It is known that in a theory with neutral vector mesons there are vertex corrections that generate a strongly damped vertex function in the ultraviolet region [8]. The damping arises from the infrared structure of the theory, despite the fact that the external nucleon momenta and the momentum transfer to the vertex are large. Physically, the damping arises from the large likelihood of matter fields to radiate soft virtual vector mesons. This phenomenon is a property of theories containing vector mesons. Based on these considerations in QED, Sudakov [9] derived a form factor. In a recent publication, Allendes and Serot [10] included the Sudakov form factor in the calculation of the polarization loop correction to the vector meson propagator. The authors concluded that inclusion of the Sudakov form factor regulates the ultraviolet behavior so that the corrected propagator is free from ghost poles. This is a very important achievement for the construction of a consistent relativistic nuclear many-body theory with mesons and baryons only, as it restores the hope for the consistency of such a theory.

In this paper we study the effect of the Sudakov and quark-substructure form factors on the ghost problem in the nucleon propagator. We consider a model with nucleons, neutral vector mesons, and pions. Although the theory is designed to study nuclear matter, we shall restrict ourselves in this paper for simplicity to the vacuum only. The appearance of the ghosts does not depend on the presence of matter [11]. We analyze the effect of the vertex corrections by means of form factors in the renormalized Schwinger-Dyson equation for the nucleon propagator. The calculation of the complete vertex function is a complicated problem and therefore we shall use simplifying approximations which we discuss below.

\section{NUCLEON PROPAGATOR AND GHOST POLES}

In order to make the paper self-contained, at the cost of being a little repetitive, in the following we briefly review the problem of ghosts in the nucleon propagator following the work of Brown, Puff, and Wilets (BPW) [3]. We start with the usual definition of the nucleon propagator

$$
G_{\beta \alpha}\left(x-x^{\prime}\right)=-i\left\langle 0\left|T\left[\psi_{\beta}\left(x^{\prime}\right) \bar{\psi}_{\alpha}(x)\right]\right| 0\right\rangle,
$$

where $\psi$ represents the nucleon field operator and $|0\rangle$ is the physical vacuum state. The Källen-Lehmann representation for the Fourier transform $G(p)$ of $G_{\beta \alpha}\left(x-x^{\prime}\right)$ can be written as

$$
G(p)=\int_{-\infty}^{+\infty} d \kappa \frac{A(\kappa)}{\not p-\kappa+i \epsilon} .
$$

$A(\kappa)$ is the spectral function. It represents the probability that a state of mass $|\kappa|$ is created by $\psi$ or $\bar{\psi}$, and as such it must be non-negative. Negative $\kappa$ corresponds to states with opposite parity to the nucleon.

Equation (2) can be rewritten conveniently as

$$
G(p)=P_{+}(p) \tilde{G}\left(w_{p}+i \epsilon\right)+P_{-}(p) \tilde{G}\left(-w_{p}-i \epsilon\right),
$$

where $P_{ \pm}(p)$ are projection operators defined as

$$
P_{ \pm}(p)=\frac{1}{2}\left(1 \pm \frac{\not p}{w_{p}}\right)
$$

with

$$
w_{p}=\sqrt{p^{2}}= \begin{cases}\sqrt{p^{2}} & \text { if } p^{2}>0 \\ i \sqrt{-p^{2}} & \text { if } p^{2}<0\end{cases}
$$

and $\tilde{G}(z)$ is given by the dispersion integral

$$
\tilde{G}(z)=\int_{-\infty}^{+\infty} d \kappa \frac{A(\kappa)}{z-\kappa} .
$$

It follows from the commutation relations that

$$
\int_{-\infty}^{+\infty} d \kappa A(\kappa)=1
$$

The inverse of the propagator can be written in terms of the projection operators $P_{ \pm}(p)$ as

$$
G^{-1}(p)=P_{+}(p) \tilde{G}^{-1}\left(w_{p}+i \epsilon\right)+P_{-}(p) \tilde{G}^{-1}\left(-w_{p}-i \epsilon\right) .
$$

Since $A(\kappa)$ is supposed to be non-negative, it is simple to show that $\tilde{G}(z)$ can have no poles or zeros off the real axis. This is known as the Herglotz property. Now, if $\tilde{G}(z)$ possesses the Herglotz property, then so does $\tilde{G}^{-1}(z)$. This permits us to write a spectral representation for $\tilde{G}^{-1}(z)$,

$$
\tilde{G}^{-1}(z)=z-M_{0}-\int_{-\infty}^{+\infty} d \kappa \frac{T(\kappa)}{z-\kappa} .
$$

$\tilde{G}^{-1}(z)$ has the Herglotz property only if $T(\kappa)$ is nonnegative.

In general, the integral in Eq. (9) is divergent and therefore needs renormalization. The usual mass and wave-function renormalizations are performed by imposing the condition that the renormalized propagator has a pole at the physical nucleon mass $M$ with unit residue. This implies that the renormalized inverse propagator $\tilde{G}_{R}^{-1}(z)$, defined as

$$
\tilde{G}_{R}^{-1}(z) \equiv Z_{2} \tilde{G}^{-1}(z),
$$

is given by

$$
\tilde{G}_{R}^{-1}(z)=(z-M)\left[1-(z-M) \int_{-\infty}^{+\infty} d \kappa \frac{T_{R}(\kappa)}{(\kappa-M)^{2}(z-\kappa)}\right]
$$


where $T_{R}(\kappa)=Z_{2} T(\kappa)$ and

$$
Z_{2}=\left[1-\int_{-\infty}^{+\infty} d \kappa \frac{T_{R}(\kappa)}{(\kappa-M)^{2}}\right]
$$

From Eq. (10), it follows that the spectral representation for $\tilde{G}_{R}(z)$ is

$$
\tilde{G}_{R}(z)=\int_{-\infty}^{+\infty} d \kappa \frac{A_{R}(\kappa)}{z-\kappa}
$$

where $A_{R}(\kappa)=A(\kappa) / Z_{2}$. In terms of renormalized quantities, $Z_{2}$ can be written as

$$
\begin{aligned}
Z_{2} & =1-\int_{-\infty}^{+\infty} d \kappa \frac{T_{R}(\kappa)}{(\kappa-M)^{2}} \\
& =\left[\int_{-\infty}^{+\infty} d \kappa A_{R}(\kappa)\right]^{-1} .
\end{aligned}
$$

In order to compare with previous work of BPW, we now consider a model with nucleons $(\psi)$, pions $(\boldsymbol{\pi})$, and omegas $\left(\omega^{\mu}\right)$ given by the following Lagrangian density:

$$
\begin{aligned}
\mathcal{L}= & \bar{\psi}\left(i \gamma_{\mu} \partial^{\mu}-i g_{0 \pi} \gamma_{5} \tau \cdot \pi-g_{0 \omega} \gamma_{\mu} \omega^{\mu}\right) \psi-\frac{1}{4} F_{\mu \nu} F^{\mu \nu} \\
& -\frac{1}{2} m_{\omega}^{2} \omega_{\mu} \omega^{\mu}+\frac{1}{2} \partial_{\mu} \pi \cdot \partial^{\mu} \pi-\frac{1}{2} m_{\pi}^{2} \pi \cdot \pi
\end{aligned}
$$

where $F^{\mu \nu}=\partial^{\mu} \omega^{\nu}-\partial^{\nu} \omega^{\mu}$. The Schwinger-Dyson equation for the nucleon propagator, Fig. 1, is given by

$G^{-1}(p)=G_{0}^{-1}(p)+3 i g_{0 \pi}^{2} \int \frac{d^{4} q}{(2 \pi)^{4}} \gamma_{5} D_{\pi}\left(q^{2}\right) G(p-q) \Gamma_{5}(p-q, p ; q)+i g_{0 \omega}^{2} \int \frac{d^{4} q}{(2 \pi)^{4}} \gamma_{\mu} D_{\omega}^{\mu \nu}\left(q^{2}\right) G(p-q) \Gamma_{\nu}(p-q, p ; q)$

where $D_{\pi}$ and $D^{\mu \nu}$ are the $\pi$ and $\omega$ propagators and $\Gamma_{5}(p-q, p ; q)$ and $\Gamma_{\mu}(p-q, p ; q)$ are the pion-nucleon and omeganucleon vertex functions, respectively. We do not consider the vector meson tadpole contribution to the nucleon propagator since it drops out in the renormalization procedure. The Hartree-Fock (HF) approximation amounts to use the noninteracting $D_{\pi}$ and $D_{\omega}$ and the bare vertices $\Gamma_{5}(p-q, p ; q)=\gamma_{5}$ and $\Gamma_{\mu}(p-q, p ; q)=\gamma_{\mu}$ in Eq. $(17)$. In the HF approximation the set of equations to be solved self-consistently for the renormalized $A_{R}(\kappa)$ and $T_{R}(\kappa)$ is given by

$$
\begin{aligned}
& T_{R}(\kappa)=\int_{-\infty}^{+\infty} d \kappa^{\prime} K\left(\kappa, \kappa^{\prime}\right) A_{R}\left(\kappa^{\prime}\right) \\
& \tilde{G}_{R}^{-1}(\kappa(1+i \epsilon))=(\kappa-M)\left[1-(\kappa-M) \int_{-\infty}^{+\infty} d \kappa^{\prime} \frac{T_{R}\left(\kappa^{\prime}\right)}{\left(M-\kappa^{\prime}\right)^{2}\left[\kappa^{\prime}-\kappa(1+i \epsilon)\right]}\right] \\
& A_{R}(\kappa)=\delta(M-\kappa)+\left|\tilde{G}_{R}^{-1}(\kappa(1+i \epsilon))\right|^{-2} T_{R}(\kappa)
\end{aligned}
$$

where $K\left(\kappa, \kappa^{\prime}\right)$ is given by

$$
\begin{aligned}
& K\left(\kappa, \kappa^{\prime}\right)=K_{\pi}\left(\kappa, \kappa^{\prime}\right)+K_{\omega}\left(\kappa, \kappa^{\prime}\right) \\
& K_{\pi}\left(\kappa, \kappa^{\prime}\right)=3\left(\frac{g_{\pi}}{4 \pi}\right)^{2}\left[\kappa^{4}-2 \kappa^{2}\left(\kappa^{\prime 2}+m_{\pi}^{2}\right)+\left(\kappa^{\prime 2}-m_{\pi}^{2}\right)^{2}\right]^{1 / 2} \frac{1}{|\kappa|^{3}}\left[\left(\kappa-\kappa^{\prime}\right)^{2}-m_{\pi}^{2}\right] \theta\left(\kappa^{2}-\left(\left|\kappa^{\prime}\right|+m_{\pi}\right)^{2}\right) \\
& K_{\omega}\left(\kappa, \kappa^{\prime}\right)=\left(\frac{g_{\omega}}{4 \pi}\right)^{2}\left[\kappa^{4}-2 \kappa^{2}\left(\kappa^{\prime 2}+m_{\omega}^{2}\right)+\left(\kappa^{\prime 2}-m_{\omega}^{2}\right)^{2}\right]^{1 / 2} \frac{1}{|\kappa|^{3}}\left[\left(\kappa-\kappa^{\prime}\right)^{2}-2 \kappa \kappa^{\prime}-m_{\omega}^{2}\right] \theta\left(\kappa^{2}-\left(\left|\kappa^{\prime}\right|+m_{\pi}\right)^{2}\right)
\end{aligned}
$$

In the above equations, $g_{\pi}$ and $g_{\omega}$ are the renormalized coupling constants, defined as $g_{\pi}=Z_{2} g_{0 \pi}$ and $g_{\omega}=Z_{2} g_{0 \omega}$.

Following BPW, let us initially consider the pion, neglecting for the moment the vector meson. Equations (18)-(20) were solved numerically by iteration, beginning in Eq. (18) with the free value for $A(\kappa), A(\kappa)=\delta(M-\kappa)$. The converged function $A(\kappa)$, for $g_{\pi}^{2} / 4 \pi=14.4$ and the

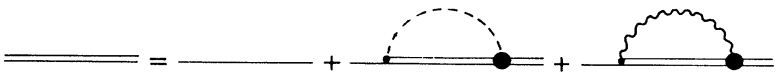

FIG. 1. Diagrammatic representation of the SchwingerDyson equation for the full nucleon propagator. The wavy (dashed) line represents the $\omega(\pi)$ meson propagator, and the solid (double solid) line represents the free (full) nucleon propagator. 
physical nucleon and pion masses, is shown by the dashed line in Fig. 2. In addition to the pole at the nucleon mass $z=M$ (which is fixed by the renormalization procedure), $\tilde{G}_{R}^{-1}(z)$ in Eq. (11) has zeros at $z=(0.73 \pm 1.25 i) M$. These complex zeroes mean that the nucleon propagator has poles at those complex masses with corresponding residues of $-0.75 \pm 0.32 i$, respectively. The signal for the presence of ghosts is revealed by the fact that $Z_{2}$ calculated from $T_{R}(\kappa)$ in Eq. (14) gives $Z_{2}=-\infty$. Since $Z_{2}^{-1}=0$, it follows from Eq. (15) that the integral of $A_{R}$ is zero. We must therefore include the pair of complex conjugated poles in $\tilde{G}_{R}$ :

$$
\tilde{G}_{R}(z)=\int_{-\infty}^{\infty} d \kappa \frac{A_{R}(\kappa)}{z-\kappa}+\frac{A_{c}}{z-\kappa_{c}}+\frac{A_{c}^{*}}{z-\kappa_{c}^{*}},
$$

where $\kappa_{c}$ and $A_{c}$ are the complex pole and residue, respectively. The sum of residues of the complex poles is negative and exactly cancels the integral over real $\kappa$. Actually, the difficulty lies in the negative sign of $Z_{2}$, since this destroys the Herglotz property of $G^{-1}$ (unrenormalized).

The ghosts have their origin in the ultraviolet behavior of the interaction, as we shall show in the following. The kernel $K_{\pi}\left(\kappa, \kappa^{\prime}\right)$ has the following asymptotic form, for large $\kappa$ or $\kappa^{\prime}$ :

$$
K_{\pi}\left(\kappa, \kappa^{\prime}\right) \longrightarrow \frac{1}{2|\kappa|^{3}}\left(\kappa^{2}-\kappa^{\prime 2}\right)\left(\kappa-\kappa^{\prime}\right)^{2} \theta\left(\kappa^{2}-\kappa^{\prime 2}\right)
$$

Since $\int d \kappa A(\kappa)$ is finite [3], it follows from Eqs. (18) and (25) that $T(\kappa)$ for large $\kappa$ is given by

$$
T_{R}(\kappa) \longrightarrow|\kappa| \text {. }
$$

Therefore, the integral in Eq. (14) is divergent and $Z_{2} \longrightarrow-\infty$.

The $\omega$ meson introduces a new ingredient in the problem, namely, the spectral function $A_{R}(\kappa)$ can be negative for some values of real $\kappa$. The dotted line in Fig. 2 represents $A_{R}(\kappa)$ for $g_{\omega}^{2} / 4 \pi=6.36$ and $m_{\omega}=780 \mathrm{MeV}$. The complex poles are located at $z=(5.67 \pm 11.76 i) M$, with

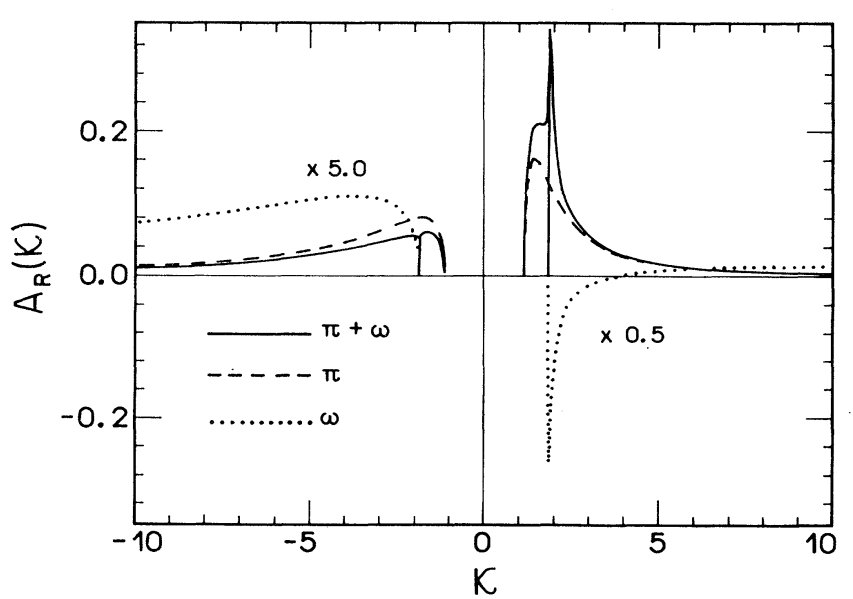

FIG. 2. Spectral function $A_{R}(\kappa)$ for $\pi$ (dashed), $\omega$ (dotted), and $\pi+\omega$ (solid). residues $-1.0412 \pm 0.22 i$, respectively. $A(\kappa)$ is negative for $M+m_{\omega} \leq \kappa<3.9$. $K_{\omega}$ has a finite negative jump at $\kappa=M+m_{\omega}$ due to the term $-2 \kappa \kappa^{\prime}$ in Eq. (23). This introduces a discontinuity in the integrand of Eq. (19) for $\tilde{G}_{R}^{-1}$. At the discontinuity, the real part (principal value integral) in Eq. (11) has a logarithmic singularity, implying that $A_{R}(\kappa)$ has a (sharp) zero at $\kappa=M+m_{\omega}$. This zero is represented in Fig. 2 by the vertical straight line which hits the $\kappa$ axis at the discontinuity.

Although negative $A_{R}(\kappa)$ represents presumably the presence of negative metric states (sometimes also referred to as ghosts), this is not related to the complex ghost poles which motivated this work. We show below that the use of form factors will eliminate the complex poles, but $A_{R}(\kappa)$ may still contain negative regions as before. For QED, the interaction kernel is the same as in Eq. (23), with $m_{\omega}=0$, giving rise to a negative spectral function as well [12]. In theories with massless vector bosons, the theory is formulated in terms of an indefinite metric [13] and the positivity of the spectral functions is not a necessary requirement [12]. However, in our case where $m_{\omega} \neq 0, A_{R}(\kappa) \geq 0$ is a necessary requirement and the only explanation we have at the moment for $A_{R}(\kappa)<0$ is the inadequacy of the HF approximation, or the inconsistency of the theory.

Including both $\pi$ and $\omega$ mesons, the situation is qualitatively similar as above. The contribution of the $\pi$ dominates the one of the $\omega$ and $A_{R}(\kappa)$ is non-negative for real $\kappa$. In Fig. 2, the solid line indicates the function $A_{R}(\kappa)$ for the same values of $g_{\pi}, m_{\pi}, g_{\omega}$, and $m_{\omega}$ as above. The complex poles are located at $z=(1.05 \mp 1.26 i) M$ with residues $-0.77 \pm 0.20 i$, respectively.

\section{VERTEX CORRECTIONS}

In this section we discuss the effect of form factors on the problem of ghost poles. We start with the consideration of the Sudakov form factor.

Let us consider the proper $N N \omega$ vertex $\Gamma^{\mu}\left(p_{1}, p_{2}, q\right)$, Fig. 3(a). The $p_{1}$ and $p_{2}$ are the external nucleon momenta and $q$ is the external meson momentum. The Sudakov form factor is obtained by summing the leading$\log$ contributions of all vertex corrections. For QED, for off-shell spacelike nucleon momenta, the Sudakov form

(a)

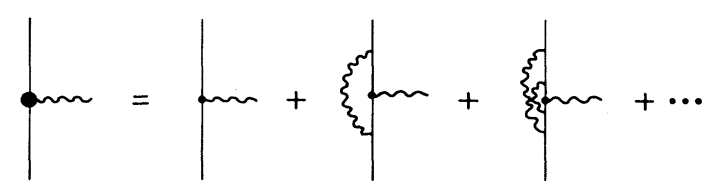

(b)

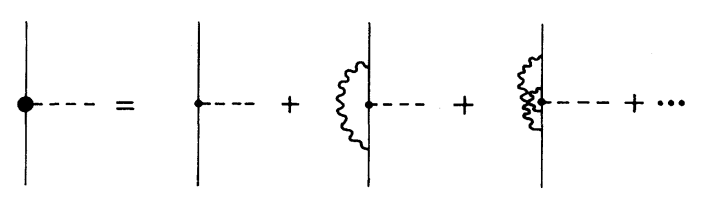

FIG. 3. Diagrammatic representation of vertex corrections for the (a) $N N \omega$ vertex and (b) $N N \pi$ vertex. 
factor is given by

$$
\Gamma^{\mu}\left(p_{1}, p_{2}, q\right)=\gamma^{\mu} \exp \left[-\frac{e^{2}}{8 \pi^{2}} \ln \left|\frac{q^{2}}{p_{1}^{2}}\right| \ln \left|\frac{q^{2}}{p_{2}^{2}}\right|\right] .
$$

This expression is valid for large nucleon momenta, $\left|p_{1}^{2}\right|$, $\left|p_{2}^{2}\right| \gg M^{2}$, and $\left|q^{2}\right| \gg\left|p_{1}^{2}\right|,\left|p_{2}^{2}\right|$. Although the momenta appearing in Eq. (27) are spacelike, Sullivan and Fishbane [14] argue that the expression may be freely continued to the timelike region. In the case of massive vector mesons, we have exactly the same expression, for large momenta, as in Eq. (27), with $e^{2}$ replaced by $g_{\omega}^{2}$.

Our approach consists in replacing the bare vertices by the corresponding vector meson corrected ones. Of course, all values of the loop momentum $q^{2}$ are formally required in the evaluation of $\tilde{G}^{-1}(p)$ in Eq. (17), corresponding to the exchanged mesons in Fig. 1. The lowest-order (BPW) approximation, using the HartreeFock form of the $\Gamma$ 's, is correct only for $q=0$ and is not asymptotically correct for high $q^{2}\left(\Gamma_{\mu}\right.$ and $\Gamma_{5}$ are entirely independent of $q$ in BPW). Since the Sudakov form, Eq. (27), introduces convergence at high momenta, one might hope that our approach will eliminate the ghost problem appearing in the ultraviolet. Although we will see that this is the case, a fully satisfactory analysis requires knowing that the asymptotic high $q^{2}$ behavior of the Sudakov form is correct.

Although the integral in Eq. (18) requires all values of $q$, a correct high $q$ behavior, together with a correct $q=0$ limit, should provide at least a "ghost-free" result. Allendes and Serot calculated in first-order perturbation theory the (low $q^{2}$ ) on-shell vertex function and then interpolated the result to the on-shell Sudakov form factor in the ultraviolet (high $q^{2}$ ). Since the calculation of the low momentum behavior of the off-shell vertex function is a tremendous task, even in lowest-order perturbation theory, we prefer not to follow the approach of Allendes and Serot. We, instead, rewrite Eq. (27) as

$$
\Gamma^{\mu}\left(p_{1}, p_{2}, q\right)=\gamma^{\mu} F\left(p_{1}, p_{2}, q\right)
$$

with

$$
F\left(p_{1}, p_{2}, q\right)=\exp \left[-\frac{g_{\omega}^{2}}{8 \pi^{2}} \ln \left|\frac{\Lambda^{2}+q^{2}}{\Lambda^{2}+p_{1}^{2}}\right| \ln \left|\frac{\Lambda^{2}+q^{2}}{\Lambda^{2}+p_{2}^{2}}\right|\right]
$$

where $\Lambda$ is an infrared cutoff which will be fixed later. The effect of $\Lambda$ is to extend the validity of form factor to the infrared region of the loop integral. The important momentum dependence of the form factor for the elimination of the ghosts is the $p_{1}$ (or $p_{2}$ ) dependence. We note that the region of large $q^{2}$ does not contribute in the loop integral, and so the conditions for the validity of the form factor in the ultraviolet region of the loop integral are satisfied in practice.

It is not difficult to show, following Sudakov's derivation [9], that the vector meson correction to the proper $N N \pi$ vertex, Fig. 3(b), gives the same exponential suppression as given by Eq. (27). We write the $N N \pi$ vertex function as

$$
\Gamma_{5}\left(p_{1}, p_{2}, q\right)=\gamma_{5} F\left(p_{1}, p_{2}, q\right),
$$

where we have also introduced an infrared regulator. With the introduction of the infrared regulator, the vertex functions have the correct zero momentum limit $F(0,0,0)=1$.

By substituting the Sudakov corrected vertex functions in the Schwinger-Dyson equation, Eq. (17), we obtain the following expression for the Sudakov corrected kernels:

$$
K_{\pi}^{\mathrm{Sud}}\left(\kappa, \kappa^{\prime}\right)=K_{\pi}\left(\kappa, \kappa^{\prime}\right) F\left(\kappa, \kappa^{\prime}, m_{\pi}\right)
$$

and

$$
K_{\omega}^{\mathrm{Sud}}\left(\kappa, \kappa^{\prime}\right)=K_{\omega}\left(\kappa, \kappa^{\prime}\right) F\left(\kappa, \kappa^{\prime}, m_{\omega}\right),
$$

where $K_{\pi}\left(\kappa, \kappa^{\prime}\right)$ and $K_{\omega}\left(\kappa, \kappa^{\prime}\right)$ are the "bare" interaction kernels of Eqs. (22) and (23).

We have also studied the ghost problem in the context of purely parametrized form factors, similar to the ones employed in boson exchange nucleon-nucleon potential models [15]. There is one complication in our case, namely, we need off-shell form factors, since the external nucleon legs are off shell. Here we follow Ref. [16] and use one suppression factor for each external leg of the vertex

$$
F\left(p_{1}, p_{2}, q\right)=\frac{1}{1+\left|p_{1}^{2} / \Lambda^{2}\right|} \frac{1}{1+\left|q^{2} / \Lambda^{2}\right|} \frac{1}{1+\left|p_{2}^{2} / \Lambda^{2}\right|}
$$

where the value of $\Lambda$ is discussed in the next section.

Substitution of this in the Schwinger-Dyson equation, Eq. (17), we obtain Eqs. (31) and (32) for the corrected kernels.

\section{NUMERICAL RESULTS}

We start discussing the Sudakov suppression for the pion. The iterative solution of the equation proceeds as before. We find that the ghost poles disappear for values of $\Lambda$ smaller than a critical value $\Lambda_{\text {crit }} \approx 0.9 M$. The function $A_{R}(\kappa)$ is shown by the dashed line in Fig. 4 for

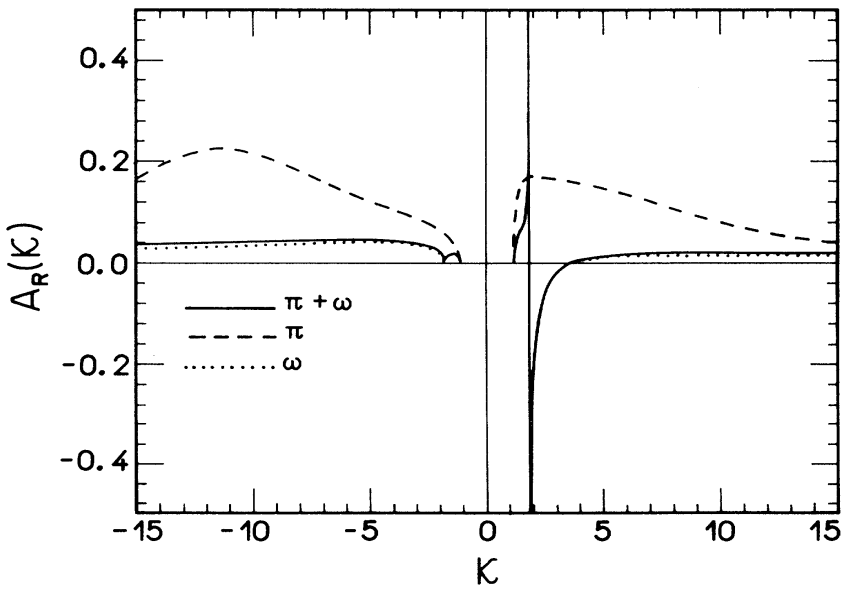

FIG. 4. Spectral function $A_{R}(\kappa)$ including the Sudakov form factor. The meaning of the different lines is the same as in Fig. 2. For values of $\Lambda$ see text. 
$\Lambda=\Lambda_{\text {crit }}$. Besides the fact that we do not find complex poles, we also find that

$$
\begin{aligned}
Z_{2} & =1-\int_{-\infty}^{+\infty} d \kappa \frac{T_{R}(\kappa)}{(\kappa-M)^{2}} \\
& =\left[\int_{-\infty}^{+\infty} d \kappa A_{R}(\kappa)\right]^{-1}=0.16 .
\end{aligned}
$$

The important point about Eq. (34) is that we get a positive $Z_{2}$, and so we do not have the usual sign for the presence of ghosts. Moreover, we obtain the same value for $Z_{2}$ calculated either with $A_{R}(\kappa)$ or with $T_{R}(\kappa)$, meaning that we do not have missing strength and so, $\tilde{G}_{R}^{-1}(z)$ given by Eq. (11) is the inverse of $G_{R}$ given by Eq. (13) [3].

For $\omega$, we find that for $\Lambda_{\text {crit }} \approx 0.55 M$, the ghosts disappear. The dashed line in Fig. 4 represents the function $A_{R}(\kappa)$ for $\Lambda=0.55 M$. Figure 4 shows that, as mentioned in Sec. II, although the ghosts poles have disappeared, $A_{R}(\kappa)$ contains a negative region as before. The obvious conclusion is that the negative metric states are not related to the BPW ghost poles.

Including both $\pi$ and $\omega$ mesons, the situation is qualitatively similar as for the $\pi$ and $\omega$ in isolation. The solid curve in Fig. 4 refers to $\Lambda=0.6 M$ for $\pi$ and $\Lambda=0.55 M$. The different values for $\Lambda_{\text {crit }}$ for $\pi$ and $\omega$ are understood on the basis of their range in the $N N$ force. This is clearly manifest in combining the case of $\pi$ and $\omega$, where the suppression is governed entirely by $\Lambda$ close to $\Lambda_{\text {crit }}$ for $\omega$.

In Fig. 5 we present the results for the spectral function when using the monopole corrected vertices of Eq. (33). The situation is similar to the Sudakov suppression with respect to the role of the cutoff and the disappearance of the ghost poles. For $\Lambda$ 's smaller than a critical value $\Lambda_{\text {crit }}$, the complex poles disappear and $Z_{2}$ calculated via $A_{R}$ and $T_{R}$ give identical results. For the plots in Fig. 5 we have used $\Lambda=M$ for all cases. In Fig. 6 , we illustrate

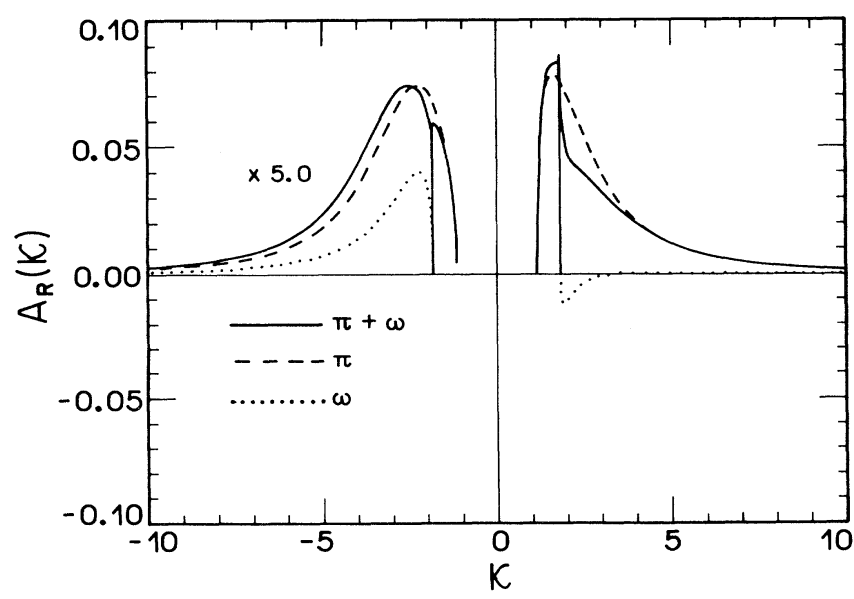

FIG. 5. Spectral function $A_{R}(\kappa)$ including the monopole form factors. The meaning of the different lines is the same as in Fig. 4. For values of $\Lambda$ see text.

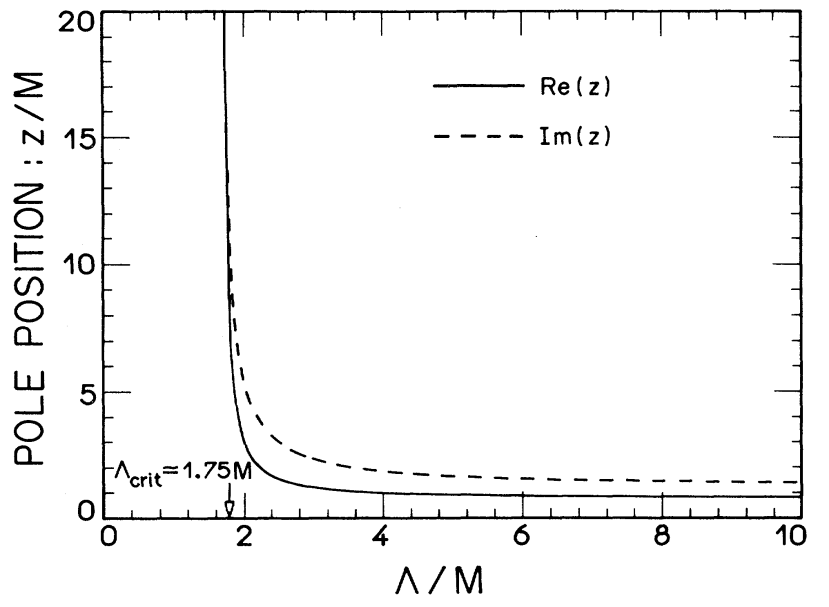

FIG. 6. Trajectory of the real (solid) and imaginary (dashed) parts of the upper complex pole of the nucleon propagator for the $\pi$ contribution as a function of the cutoff parameter in the monopole form factors. The arrow indicates the critical value of $\Lambda$ for the disappearance of the ghost poles.

the trajectory of the complex poles as a function of $\Lambda$, for the case of $\pi$. The critical value of $\Lambda$ for the disappearance of the ghosts is of the same order (Fig. 6) as used in potential models [15]. One interesting feature of the different form factors is that the Sudakov form factor "squeezes" the spectral function around $\kappa=M+m_{\omega}$, while the monopole form factor has the effect of decreasing the spectral function all over the $\kappa$ range. This is possibly due to the fact that the Sudakov form factor provides a strong suppression for high momenta only, while the monopole form factors provide a uniform suppression starting at momenta of the order of the nucleon mass.

\section{CONCLUSIONS}

The nucleon propagator treated in the Hartree-Fock approximation in a hadronic field theory is plagued with the presence of ghost poles. The appearance of the ghosts is related to the ultraviolet behavior of the nucleon-meson interaction. In a theory containing vector mesons, the infrared structure of such a theory introduces corrections to the various nucleon-meson vertex functions which are strongly damped in the ultraviolet. The damping arises from the dressing of these vertices by the vector mesons. In this work we have shown that such a damping of the vertex function can eliminate the appearance of the ghost poles of the usual Hartree-Fock approximation.

We emphasize that our work has to be extended and improved on several aspects before definite conclusions can be drawn for realistic calculations in nuclear physics. First, although the ghost problem has been shown not to be related to the presence of a Fermi sea, the effects of the Sudakov form factor in this case is worth investigating. In our work we have simply used the Sudakov form factor in the loop integral of the Schwinger-Dyson equation. The 
off-shell behavior of the vertex function at low $q^{2}$ has to be investigated.

In summary, we have shown that the inclusion of corrections due to the dressing of vector mesons of nucleon- meson vertex functions are able to soften the ull aviviec behavior of hadronic field theories. Therefore, vacuum corrections to mean field approximations can be calculated in a consistent way.
[1] J.D. Walecka, Ann. Phys. (N.Y.) 83, 491 (1974); L.S. Celenza and C.M. Shakin, Relativistic Nuclear Physics: Theories of Structure and Scattering (World Scientific, Singapore, 1986).

[2] P.A.M. Dirac, Proc. R. Soc. London, Ser. A 180, 1 (1942); W. Pauli and F. Villars, Rev. Mod. Phys. 15, 175 (1943); 21, 21 (1949); T.D. Lee, Phys. Rev. 95, 1329 (1954); E.C. Sudarshan, Proceedings of the 14th Solvay Conference, Brussels, Belgium, 1967 (Interscience, New York, 1968).

[3] W.D. Brown, R.D. Puff, and L. Wilets, Phys. Rev. C 2, 331 (1970); L. Wilets, in Mesons in Nuclei, edited by M. Rho and D. Wilkinson (North-Holland, Amsterdam, 1979).

[4] R.J. Perry, Phys. Lett. B 199, 489 (1987); T.D. Cohen, M.K. Banerjee, and C.-Y. Ren, Phys. Rev. C 36, 1653 (1987); K. Wehrberger, R. Wittman, and B.D. Serot, ibid. 42, 2680 (1990).

[5] J. Achtzehnter and L. Wilets, Phys. Rev. C 38, 5 (1988).

[6] P.J. Redmond, Phys. Rev. 112, 1404 (1958); P.J. Redmond and J.L. Uretsky, Phys. Rev. Lett. 1, 147 (1958); K. Tanaka, W. Bentz, and A. Arima, Nucl. Phys. A518,
229 (1990).

[7] M. Prakash, P.J. Ellis, and J.I. Kapusta, Phys. Rev. C 45, 2518 (1992); R. Friedrich, K. Wehrberger, and F. Beck, ibid. 46, 188 (1992).

[8] G.E. Brown and A.D. Jackson, The Nucleon-Nucleon Interaction (North-Holland, Amsterdam, 1976); J. Milana, Phys. Rev. C 44, 527 (1991).

[9] V.V. Sudakov, Zh. Eksp. Teor. Fiz. 30, 87 (1956) [Sov. Phys. JETP 3, 65 (1956)].

[10] M.P. Allends and B.D. Serot, Phys. Rev. C 45, 2975 (1992).

[11] L. Wilets, R.D. Puff, D. Chiang, and W.T. Nutt, Phys. Rev. C 14, 2269 (1976).

[12] M. Gell-Mann and F.E. Low, Phys. Rev. 95, 1300 (1992).

[13] S.W. Gupta, Proc. Phys. Soc. London 63, 681 (1950); K. Bleuler, Helv. Phys. Acta 23, 567 (1950).

[14] P.M. Fishbane and J.D. Sullivan, Phys. Rev. D 4, 458 (1971).

[15] R. Machleidt, K. Holinde, and Ch. Elster, Phys. Rep. 149, 1 (1987).

[16] F. Gross, J.W. Van Orden, and K. Holinde, Phys. Rev. C 45, 2094 (1992). 